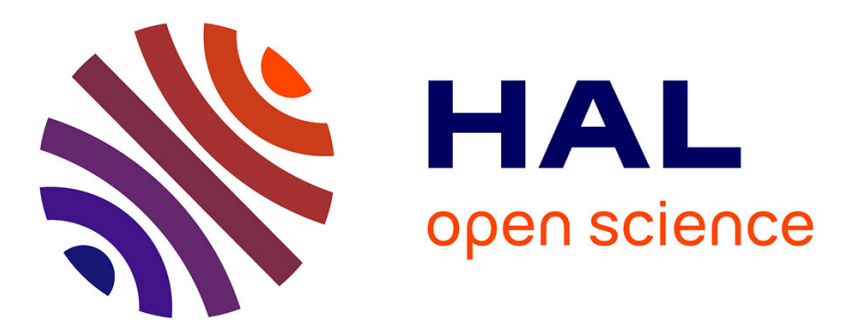

\title{
Photo-stimulated discharge current measurements on biaxially oriented polypropylene thin films (BOPP)
}

\author{
Laurent Boudou, F. Zheng, G. Teyssedre
}

\section{To cite this version:}

Laurent Boudou, F. Zheng, G. Teyssedre. Photo-stimulated discharge current measurements on biaxially oriented polypropylene thin films (BOPP). 2018 12th International Conference on the Properties and Applications of Dielectric Materials (ICPADM), May 2018, Xi'an, China. pp.722-725, 10.1109/ICPADM.2018.8401125 . hal-02396117

\section{HAL Id: hal-02396117 \\ https://hal.science/hal-02396117}

Submitted on 11 Dec 2020

HAL is a multi-disciplinary open access archive for the deposit and dissemination of scientific research documents, whether they are published or not. The documents may come from teaching and research institutions in France or abroad, or from public or private research centers.
L'archive ouverte pluridisciplinaire HAL, est destinée au dépôt et à la diffusion de documents scientifiques de niveau recherche, publiés ou non, émanant des établissements d'enseignement et de recherche français ou étrangers, des laboratoires publics ou privés. 


\title{
Photo-stimulated Discharge Current Measurements on Biaxially Oriented Polypropylene Thin Films (BOPP)
}

\author{
L. Boudou ${ }^{1}$, F. Zheng $^{2}$ and G. Teyssedre ${ }^{1}$ \\ ${ }^{1}$ Laplace, CNRS and University of Toulouse, France \\ ${ }^{2}$ Department of Electrical Engineering, Tongji University, Shanghai, China
}

\begin{abstract}
The photo-stimulated discharge method (PSD) is a very good tool to estimate charge trap energy levels in transparent or semi-transparent materials. Its advantage, when compared to thermally stimulated discharge (TSD), is to probe deeper charge traps without any thermal effect that can affect the material microstructure and the charge stability. In this work, PSD measurements were performed on biaxially oriented polypropylene films. The PSD spectrum shows two peaks located at $400 \mathrm{~nm}$ and $240 \mathrm{~nm}$ scanning light wavelength. The effect of the electrode structure (fingered vs. continuous electrodes) and the effect of applied field intensity are discussed.
\end{abstract}

\section{INTRODUCTION}

The interest of studying charge transport and trapping in dielectric materials has continuously increased over the past decades. Among the experimental methods that allow investigating charge trapping phenomena, those providing access to the distribution of trap levels are not numerous. The most used ones are probably the Thermally-stimulated discharge method (TSD) and the Photo-stimulated discharge method (PSD). TSD has the advantage to be a quick and useful method that allows to probe both dipolar processes and trapped charge release during a temperature ramp-up. Associated with space charge measurement techniques it can allow to dissociate charge detrapping processes from relaxation of orientation polarization [1]. However, the main problems of TSD concern the temperature effect on the microstructure of the material during measurement (annealing effect), the fact that charge release may correspond to charge dissipation due to destruction of the trap due to chain motion rather than release of the charge by thermal activation and that TSD is not appropriated to deep traps probing in polymer materials, because the temperature increase is limited by the melting of the tested material. In addition, the kinetic analysis of TSD glow curves is not straightforward, especially if overlapping distributed processes are at play. In the PSD method, introduced in the 1970s, the charge release is induced by irradiation of the sample with monochromatic light. This method is well adapted to estimate charge trap energy level in transparent or semi-transparent materials [2]. The progress in illuminating source development, especially in the deepultraviolet range $(200-300 \mathrm{~nm})$ allows probing deeper traps compared to PSD, without any detrimental effect on the sample microstructure. Nonetheless the interpretation of the
PSD data is sometimes difficult. Actually, the recorded PSD current can originate from space charge detrapping, but also from photoconduction effect, photoelectric effect or distorted by dipolar relaxations during measurement $[3,4]$.

The first part of this paper is dedicated to the description of the test bench including irradiation source characterization, electrode structure and experimental protocol description. In the second part the charge/discharge currents and the PSD spectra obtained on biaxially oriented polypropylene (BOPP) films for different applied voltage are presented and discussed.

\section{Methodology}

\section{A. PSD apparatus}

The circuit diagram for PSD measurement is shown in Fig 1. The monochromatic light is produced by a $150 \mathrm{~W}$ Xenon arc lamp associated with a monochromator equipped with a $300 \mathrm{~nm}$-blaze grating $(1200 \mathrm{~g} / \mathrm{mm})$. The bandwidth of the monochromatic light can be controlled adjusting the input and output slits ( $2 \mathrm{~mm}$ for both in this study). All the experiments were performed in air atmosphere and at room temperature. The output power $\mathrm{P}(\mathrm{W})$ was measured as a function of the wavelength using a thermopile sensor (ref Newport 919P-00310). Considering a monochromatic light and an irradiation spot of $3 \mathrm{~cm}$ in diameter at the sample surface, the photon flux density could be estimated, cf. Fig. 2(a). The flux is roughly constant in the $400 \mathrm{~nm}-800 \mathrm{~nm}$ range and decreases in the UV range when scanning down to $200 \mathrm{~nm}$.

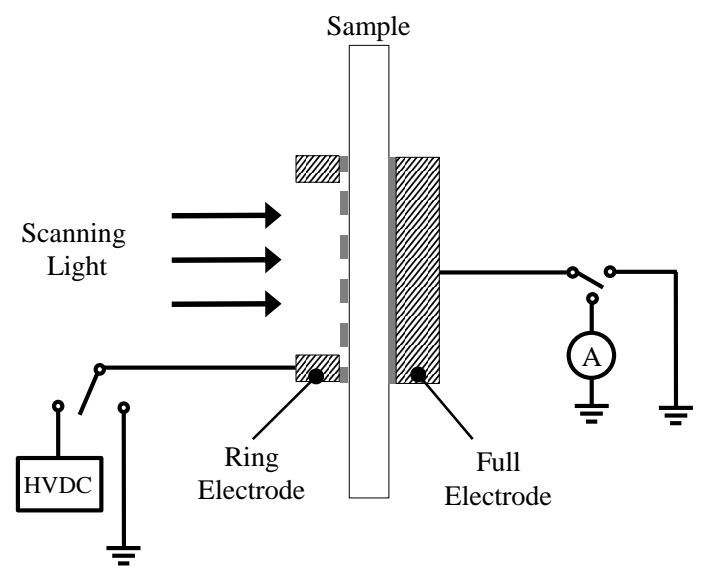

Fig. 1. PSD Circuit diagram 
In order to evaluate the wavelength bandwidth of light at the output of the monochromator, characterization at a $240 \mathrm{~nm}$ fixed wavelength was performed using a spectrometer (model HR4000-UV-NIR from Horiba). The spectrum, shown in Fig. 2(b) -see also Table 1, reveals the fundamental peak located at $240 \mathrm{~nm}$ as well as the harmonic peaks, which are not filtered in our apparatus. The bandwidth obtained using an exit slit of $2 \mathrm{~mm}$ is $7 \mathrm{~nm}$ (it can be decreased by using a more narrow slit, but this is detrimental to the output power). The harmonics produced by the monochromator have non-negligible amplitude. However, as the scanning is achieved from large to small wavelength, their contribution has no real effect on the measured PSD spectra.

The samples are previously charged under DC voltage. During conditioning, the charge and discharge current are registered using a Keithley 617 electrometer connected to the back electrode. In this configuration the polarization currents are positive (for a positive DC voltage applied), and depolarization currents are negative. For clarity, the PSD

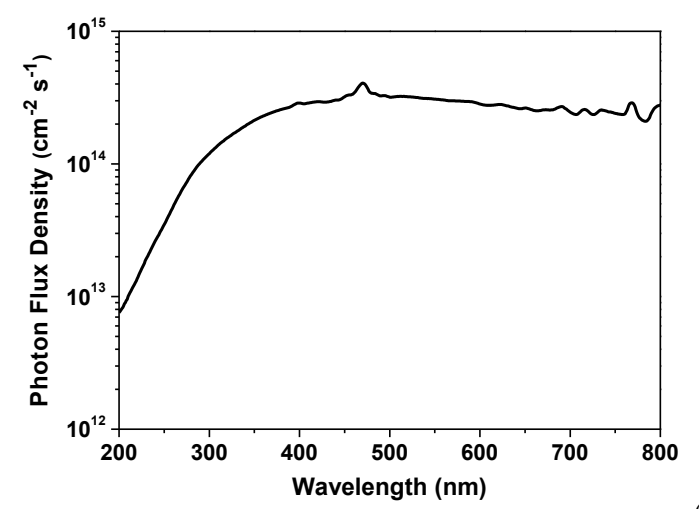

(a)

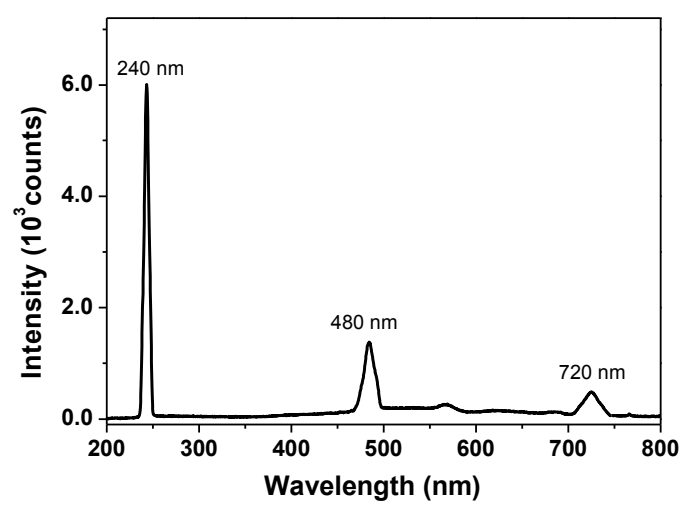

(b)

Fig. 2. Photon flux density versus light wavelength (a) and $240 \mathrm{~nm}-$ illuminating light characteristic

TABLE 1.

CHARACTERISTIC OF THE MONOCHROMATOR FOR A SELECTED WAVELENGTH OF $240 \mathrm{NM}$.

\begin{tabular}{|c|c|c|c|}
\hline & Fundamental & $\begin{array}{c}\text { Harmonic } \\
\mathbf{n = 2}\end{array}$ & $\begin{array}{c}\text { Harmonic } \\
\mathbf{n = 3}\end{array}$ \\
\hline Peak w.l. (nm) & 240 & 480 & 720 \\
\hline Imax (counts) & 6638 & 2001 & 1112 \\
\hline FWHM (nm)* & 7 & 14 & 21 \\
\hline
\end{tabular}

*Full width at half Maximum spectra are presented in positive values, taking the opposite sign of the PSD current recorded during the measurement.

\section{B. Material}

The material used in this study is a bi-oriented polypropylene (BOPP) film of $18 \mu \mathrm{m}$ in thickness provided by Kopafilm, Germany. The films are provided with rough surfaces to promote impregnation of capacitors. Test samples were BOPP discs of $8 \mathrm{~cm}$ in diameter, with gold electrode sputtered on each face. For the electrode facing to the scanning light (termed as the front side), we used a fingered electrode having a diameter of $4 \mathrm{~cm}$ (Fig. 3) with a thickness of $50 \mathrm{~nm}$. For the electrode connected to the ammeter (termed back electrode), we used a sputtered full gold electrode with a thickness of $50 \mathrm{~nm}$ from measurements on gold layers processed with the same sputtering equipment, the transmittance of a $50 \mathrm{~nm}$-gold layer is estimated to less than $30 \%$ [5] in the UV wavelength range.

\section{Measurement Protocol}

The experimental protocol used is represented in Fig. 4. The samples were polarized at constant voltage $(1 \mathrm{kV}, 2 \mathrm{kV}$ or $3 \mathrm{kV}$ corresponding to electrical fields of $55 \mathrm{kV} / \mathrm{mm}, 110$ $\mathrm{kV} / \mathrm{mm}$ and $167 \mathrm{kV} / \mathrm{mm}$ respectively) during $30 \mathrm{~min}$. After the polarization step the sample was short-circuited during 30 min. before the light irradiation starts and the discharge current was measured. The short circuit step is to complete fast relaxation processes in order for the PSD spectrum to be initiated with a negligible current. The PSD current is recorded during the light scanning from low energy $(590 \mathrm{~nm})$

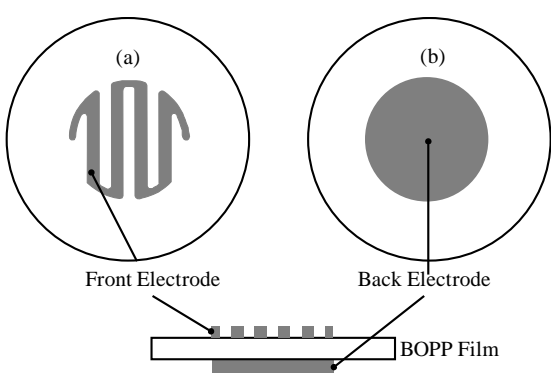

Fig. 3. Electrode structure. (a) Finger electrode (b) Full electrode

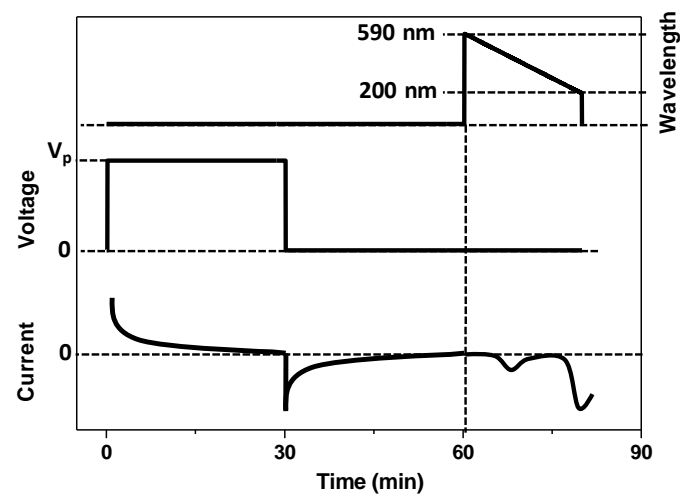

Fig. 4. Experimental protocol 
to high energy $(200 \mathrm{~nm})$ with a scanning rate of $24 \mathrm{~nm} / \mathrm{min}$. Scanning at longer wavelength did not produce significant current and could possibly heat the sample.

\section{RESULTS AND DISCUSSION}

\section{A. Polarization and depolarization current}

Fig. 5 (a) shows the transient current registered during the polarization step, for different applied voltages. It shows that the steady-state current is not reached after 30 minutes of polarization. It is then difficult to make use of the current versus voltage characteristic in order to identify the conduction mechanism at play in the field range explored.

The threshold field between ohmic and nonlinear behavior can be estimated at about $40 \mathrm{kV} / \mathrm{mm}$ from charging currents measured over $24 \mathrm{~h} \mathrm{[6]}$. With using shorter voltage steps of 5 min, a steeper increase of the current occurs beyond $100 \mathrm{kV} / \mathrm{mm}$ and weak electroluminescence was detected for fields beyond $200 \mathrm{kV} / \mathrm{mm}$ under DC stress [7], meaning that there is not a great amount of charge recombination events. This is consistent with the fact BOPP is known to be highly resistive. In the field range investigates herein, 50 to 150 $\mathrm{kV} / \mathrm{mm}$, charge injection from the electrodes and trapping within the polymer bulk are probably the main mechanisms at play. Furthermore, Fig. 5(b) shows that there is no superposition when polarization and depolarization current are compared, even at the lower applied field, meaning that the contribution from dipolar mechanisms to the registered current is negligible in the invstigated field range.
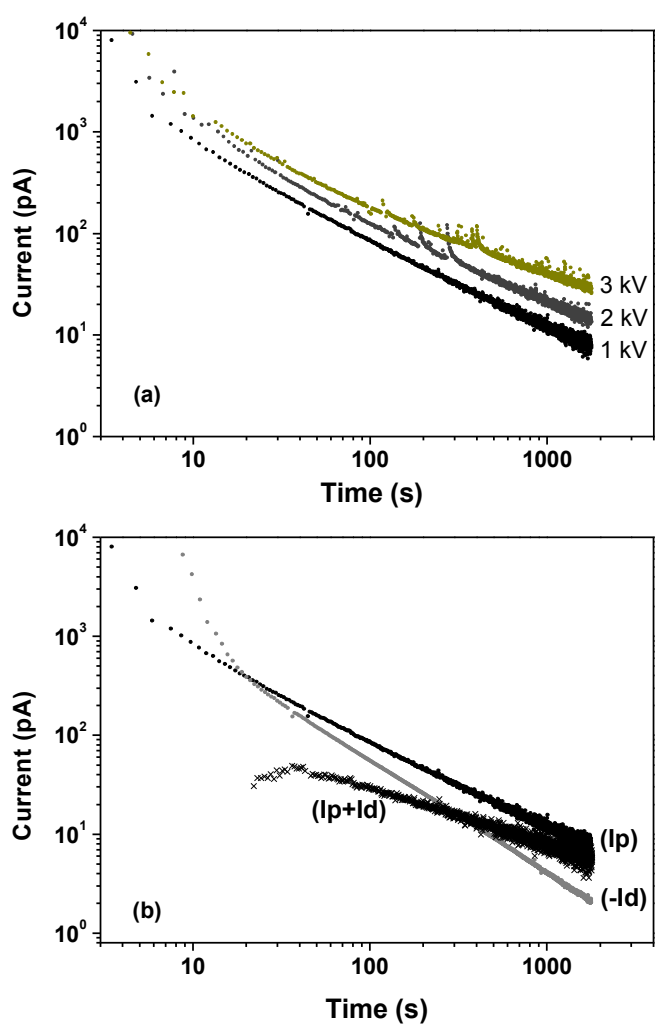

Fig. 5 (a) Transient current recorded during polarization step for $1 \mathrm{kV}$, $2 \mathrm{kV}$ and $3 \mathrm{kV}$ applied voltage and (b) Polarization current (Ip) and depolarization current (-Id) for $1 \mathrm{kV}$ applied voltage.

\section{B. PSD spectra}

The blank PSD spectrum, performed without any stress previously applied to the sample, is flat, cf. Fig .6. When using a full semi-transparent Au electrode with a thickness of $18 \mathrm{~nm}$ facing to the light beam, the PSD spectrum show two peaks (results not shown here) located at around $220 \mathrm{~nm}$ and 400 $\mathrm{nm}$, but their amplitudes are small, even at high voltage. In order to improve the signal-to-noise ratio, the incident light intensity can be increased by increasing the width of input and output slits, with a loss of resolution in wavelength. Another way is to use a fingered electrode facing to the irradiation light. The current signal is higher because of higher light penetration within the material bulk. On the other hand, one of the indirect effects of using such fingered electrode is that electric field is enhanced locally due to edge effects, and as a consequence charge injection and trapping at these "hot points" can be substantially increased [8].

On the PSD spectra shown in Fig.6, two current peaks are observed, located at approximatively at $400 \mathrm{~nm}$ and $220 \mathrm{~nm}$. The peaks are superimposed to a baseline current, voltage dependent, which is in the continuity of the transient current curves shown in Fig. 5a. Note here that the PSD spectra were not corrected for the output power of the monochromator. Considering Fig.2a, The amplitude of the $220 \mathrm{~nm}$ peak would be magnified by at least one decade in respect to the peak at $400 \mathrm{~nm}$ after such correction. The PSD intensity increases with the voltage applied during the polarization step increases, the intensity of the peak located at $220 \mathrm{~nm}$ being higher when compared to the peak located at $400 \mathrm{~nm}$. One notices that the intensity is very weak for $1 \mathrm{kV}$ polarization; it increases substantially for 2 and $3 \mathrm{kV}$, revealing that the threshold for massive charge injection has been reached. For both peaks, the wavelength position is not affected when the voltage is increased. Furthermore, the peak current $\Delta \mathrm{I}_{\max }$ (taken between the maximum and the baseline of the peak) does not increase linearly with the applied voltage (cf. insert of Fig. 6.). The shapes of the two recorded peaks are different. The $400 \mathrm{~nm}-$ peak is broad (spreading from $480 \mathrm{~nm}$ to $360 \mathrm{~nm}$ with a FWHM of $50 \mathrm{~nm}$ ) and asymmetric. The $220 \mathrm{~nm}$-peak is

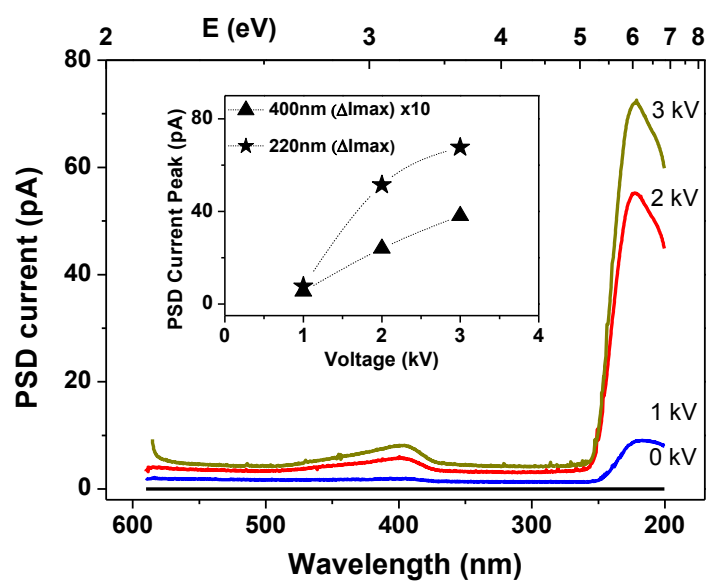

Fig. 6. PSD spectra of $18 \mu$ m-thick BOPP recorded after polarisation process under voltage of $1 \mathrm{kV}, 2 \mathrm{kV}$ and $3 \mathrm{kV}$, using fingered electrode as front electrode. Inset: voltage dependence of PSD peaks. 
narrower and asymmetric. The rate of the current increase with decreasing wavelength is also different when the two peaks are compared, and estimated at $2.74 \mathrm{pA} / \mathrm{nm}$ for the $220 \mathrm{~nm}$-peak and $0.045 \mathrm{pA} / \mathrm{nm}$ for the $400 \mathrm{~nm}$-peak for the PSD spectrum obtained after polarization under $3 \mathrm{kV}$. These differences are indications that the two peaks originate from different mechanisms.

The $220 \mathrm{~nm}$-peak is located in a wavelength range wealthy in optical signature when considering the UV-absorption spectrum of BOPP measured at room temperature [9]. This spectrum shows an absorption maximum at $200 \mathrm{~nm}$ and a first shoulder at $230 \mathrm{~nm}$, typical of $\pi \rightarrow \pi *$ transitions in $\mathrm{C}=\mathrm{C}$ double bonds. A second shoulder at $280 \mathrm{~nm}$ is rather due to the $\mathrm{n} \rightarrow \pi *$ transition in carbonyl compounds. These two regions (230 and $280 \mathrm{~nm}$ ) are those providing maximum in photoluminescence of polyolefins [9]. The $400 \mathrm{~nm}$-peak does not correspond to known absorption band in polyolefins in general, neither of the BOPP investigated herein. Either it corresponds to some additive that could efficiently trap charges, or possibly to localized states formed under the effect of space charge [10]. Another feature not to neglect is the possibility that electrons be generated from the illuminated electrode by photo-generation [8]. It is difficult at the present stage to identify the physical and/or chemical origin of the traps responsible of the PSD signature of BOPP film without any further investigations. A further point to address is the large difference in energies obtained by PSD (over $2 \mathrm{eV}$ ) compared to TSD (of the order of $1 \mathrm{eV}$ ) or for charge detrapping in polyolefins [11].

\section{Photo-discharge transient current}

Another way of analyzing the discharge consists in measuring the current decay as a function of time under fixed wavelength. Fig. 7 shows the result obtained with charging under $3 \mathrm{kV}$ with the same protocol as previously and following the decay. The current shows a fast drop in the first minutes followed by a much slower decay lasting for several hours after the irradiation light on. Charges released probably follow multiple trapping on their flow back to the electrodes (or same for photo-generated carriers to drift into the bulk). This means that during scanning procedure used in the classical PSD method, the detrapping of charges is not complete for a given

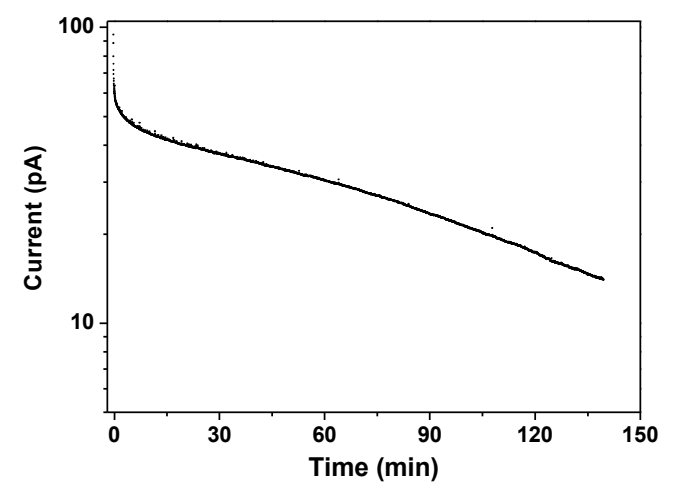

Fig. 7. Transient photodischarge current under continuous illumination at $220 \mathrm{~nm}$. Sample previously polarized under $3 \mathrm{kV}$ for $30 \mathrm{~min}$. wavelength. This could bring some confusion when PSD spectra are analyzed.

\section{CONCLUSIONS}

The use of fingered electrode as front electrode for PSD method allows to increase the current intensity without loss of wavelength resolution. Injection and trapping phenomena are enhanced due to edge effects but the local electric field value is difficult to control in this configuration. The PSD spectrum of $18 \mu \mathrm{m}$ thick BOPP film shows two peaks located at $400 \mathrm{~nm}$ and $240 \mathrm{~nm}$ scanning light wavelength. The blank PSD being flat, the PSD current recorded in short-circuit conditions is most probably linked to charge detrapping phenomena. However, the interpretation of the peak wavelength positions is not trivial and further work is needed to identify the physical and/or chemical origin of the traps responsible of the PSD signature of BOPP film.

\section{ACKNOWLEDGEMENT}

The research is supported by the program IDEX Actions Thématiques Stratégiques ATS 2015 of the Université de Toulouse under project SEPHIR (2016-066-CIF-D-DRVD).

\section{REFERENCES}

[1] M. Q. Hoang, L. Boudou, S. Le Roy and G. Teyssedre, "Dissociating space charge processes from orientation polarization in Poly(ethylene naphthalate) films", J. Phys. D: Appl. Phys., Vol. 47, p. 455306, 2014.

[2] A. Mellinger, F. C. Gonzalez and R. Gerhard-Multhaupt, "Photostimulated discharge in electret polymers: an alternative approach for investigating deep traps", IEEE Trans. Dielectr. Electr. Insul., vol. 11, p. 218, 2004.

[3] F. Zheng, W. Li, M. Gu, Z. An and Y. Zhang, "Photo-stimulated discharge current in polyimide films", IEEE Trans. Dielectr. Electr. Insul., vol. 24, p. 1802, 2017.

[4] L.J. He, X. Yang, H.Q. Niu, Z.H. Yuan, D.W. Li and C.T. Chen, "Influence of photoconductivity on the study of trap levels in polyimide films by photo-stimulated discharge", Modern Phys. Lett. B, vol. 30, p $1650410,2016$.

[5] B. Qiao,"Electrical ageing of insulating polymers: approach through electroluminescence and cathodoluminescence analyses, $\mathrm{PhD}$ Thesis, university of Toulouse, 2015.

[6] H. Li, Z. Li, F. Lin, D. Liu, B. Wang, Y. Chen, L. Dai and S. Fan, "Electrical conduction of metallized BOPP films based on revised Poole-Frenkel effect", J. Electrostatics, vol. 71, p.958, 2013.

[7] B. Qiao, C. Laurent and G. Teyssedre, "Evidence of exciton formation in thin polypropylene films under AC and DC fields and relationship to electrical degradation", IEEJ Trans. Fundam. Mater., vol. 136, p.74, 2016.

[8] P. Ma, Y. Zhang, S Holé, F. Zheng, M. Gua and Z. An, "Analysis and interpretation of photo-stimulated discharge spectrum for polypropylene films under different electric and geometrical conditions", $J$. Electrostatics, vol. 84, p.128, 2016.

[9] B. Qiao, G. Teyssedre and C. Laurent, "Field- and electron beaminduced luminescence phenomena in polypropylene thin films", Proc. Int. Conf. Properties and Applications of Dielectric Materials (ICPADM), Sydney, Australia, p. 196, 2015.

[10] C. B. Duke, "Optical absorption due to space-charge-induced localized states", Phys. Rev., vol. 159, p.632, 1967.

[11] Y. Suzuoki, T. Mizutani, Y. Takai and M.Ieda, "Investigation of Carrier Traps in Polyethylene by PSDC and TSC", Jpn. J. Appl. Phys., vol. 16, p. $1929,1977$. 